\title{
Tight Error Bounds for Asynchronous Multicarrier CDMA and Their Application
}

\author{
Kun-Wah Yip, Member, IEEE, and Tung-Sang Ng, Senior Member, IEEE
}

\begin{abstract}
Upper and lower bounds on the average bit error rate for binary phase-shift keying (BPSK), asynchronous multicarrier (MC)-CDMA communications using coherent detection are derived. The bounds can be made very tight by adjusting a parameter in the computation and this is demonstrated by numerical examples. Based on the derived bounds it is shown by numerical examples that the performance of asynchronous MCCDMA using Zadoff-Chu sequences is better than that using Walsh codes.
\end{abstract}

Index Terms-Error analysis, multicarrier CDMA.

\section{INTRODUCTION}

$\mathbf{M}$ ULTICARRIER (MC)-CDMA, which has been proposed for indoor wireless and cellular communications [1], [2], is a spread-spectrum transmission scheme that spreads the signal in the frequency domain. Many researchers have derived bit-error rate (BER) expressions for time-synchronous MC-CDMA communications [1]-[5]. However, those for asynchronous transmission have not appeared in the previous literature to the authors' best knowledge. In this letter, we derive upper and lower bounds on the average BER for asynchronous MC-CDMA.

The derived bounds are used to compare Walsh codes and Zadoff-Chu sequences on the performance of asynchronous MC-CDMA. This comparison is motivated by the recent work of Popović [6]. In [6], numerical results have shown that Zadoff-Chu sequences have much smaller maximum spectral cross-correlation magnitude ${ }^{1}$ than Walsh codes, implying that Zadoff-Chu sequences yield better worst-case performance than Walsh codes in asynchronous MC-CDMA. The average performance, however, is often more useful than the worstcase performance in determining the goodness of codes (for a reason, see [7]). It is thus useful to compare Walsh and Zadoff-Chu sequences based on the average performance.

\section{System Model AND ERROR BoundS}

We consider a MC-CDMA system with $K$ users asynchronously transmitting binary phase-shift keying (BPSK) signals at the same power $P$ and at the same bit transmission rate

Manuscript received May 1, 1998. The associative editor coordinating the review of this letter and approving the publication of this letter was Prof. L. Rusch. This work was supported by the Hong Kong Research Grants Council and by the University Research Committee of The University of Hong Kong, Hong Kong.

The authors are with the Department of Electrical and Electronic Engineering, The University of Hong Kong, Hong Kong (e-mail: kwyip@hkueee.hku.hk; tsng@hkueee.hku.hk).

Publisher Item Identifier S 1089-7798(98)08066-1.

${ }^{1}$ The spectral cross correlation characterizes the multiple access interference in asynchronous MC-CDMA systems [6].
$1 / T_{b}$. Although BPSK is considered here for illustration, our approach of error-bound derivation can be easily extended to higher modulation formats. The receiver is intended to detect the $L$ th-user signal. Each bit is transmitted on $N$ subcarriers with adjacent subcarrier spacing $\Delta f$, and each subcarrier is further modulated by the corresponding chip of a spreading sequence. For illustration purpose, we assume that the subcarriers are spectrally disjoint. We consider an additive white Gaussian noise channel with two-sided power spectral density $N_{0} / 2$. The complex envelope of the received signal $r(t)$ is given by

$r(t)=n(t)+\sum_{k=1}^{K} \sqrt{2 P} e^{j \theta_{k}} \sum_{n=-\infty}^{\infty} b_{n}^{(k)} \phi_{k}\left(t-n T_{b}-\tau_{k}\right)$

where $n(t)$ is the complex Gaussian noise, $\theta_{k}$ is the phase of the $k$ th-user signal modeled by a uniform random variable over $[0,2 \pi), b_{n}^{(k)} \in\{+1,-1\}$ is the $n$th bit of the $k$ th-user data stream, $\tau_{k}$ is the delay of the $k$ th-user signal relative to the Lth-user signal, and

$$
\phi_{k}(t)=\frac{1}{\sqrt{N}} \psi(t) \sum_{m=0}^{N-1} c_{m}^{(k)} e^{j 2 \pi m(\Delta f) t} .
$$

In (2), $\psi(t)$ is the pulse shape satisfying $\int_{-\infty}^{\infty}|\psi(t)|^{2} d t=T_{b}$, and $c_{m}^{(k)}$, where $\left|c_{m}^{(k)}\right|=1$, is the $m$ th chip of the $k$ thuser spreading sequence. We model that $\tau_{L}=0$ and that $\tau_{k}$ 's, $k \neq L$, are independent uniform random variables over $\left[-0.5 T_{b}, 0.5 T_{b}\right]$. The received signal is processed by a matched filter that coherently detects the $L$ th-user signal. Without loss of generality we consider detection of $b_{0}^{(L)}$ and assume that $b_{0}^{(L)}=1$. The detector output, $\hat{b}_{0}^{(L)}$, is given by

$$
\hat{b}_{0}^{(L)}=\left(2 E_{b} T_{b}\right)^{-1 / 2} \operatorname{Re}\left\{\int_{-\infty}^{\infty} r(t) e^{-j \theta_{L}} \phi_{L}^{*}(t) d t\right\}
$$

where $E_{b}=P T_{b}$ is the bit energy. Substituting (1) into (3) yields

$$
\hat{b}_{0}^{(L)}=\tilde{n}+b_{0}^{(L)}+\sum_{k=1, k \neq L}^{K} J_{k}
$$

where $\tilde{n}$ is a zero-mean Gaussian random variable with variance $\left(2 E_{b} / N_{0}\right)^{-1}$, and

$$
\begin{aligned}
J_{k}=\operatorname{Re}\left\{e^{j\left(\theta_{k}-\theta_{L}\right)}\right. & \sum_{n=-n_{s}}^{n_{s}} b_{n}^{(k)} \chi_{k, L} \\
& \left.\left(\left(n T_{b}+\tau_{k}\right) \Delta f\right) \cdot R\left(n T_{b}+\tau_{k}\right)\right\}
\end{aligned}
$$


In $(5)$,

$$
\begin{aligned}
\chi_{k, L}(\mu) & =\frac{1}{N} \sum_{m=0}^{N-1} c_{m}^{(k)} c_{m}^{(L) *} e^{-j 2 \pi m \mu} \\
R(\tau) & =\frac{1}{T_{b}} \int_{-\infty}^{\infty} \psi(t) \psi^{*}(t+\tau) d t
\end{aligned}
$$

and $n_{s}$ is chosen such that $R\left(n T_{b}+\tau_{k}\right)$ is negligible when $|n|>n_{s}$. Note that (5) is derived under the assumption that subcarriers are spectrally disjoint so that intercarrier interference needs not be taken into consideration. In the presence of spectral overlapping, one may modify (5) to include intercarrier interference.

Based on the technique adapted from [8], bounds on the average bit-error rate (BER) are derived as follows. Since $J_{k}$ is expressed in terms of random variables $\theta_{k}$, etc., it follows that $J_{k}$ is also a random variable having a probability distribution function (PDF). By dividing random variables $\theta_{k}$, etc., in small step size over their ranges and by means of counting, one can obtain an approximate PDF of $J_{k}$ :

$$
\begin{aligned}
\operatorname{Pr}\left\{\left(m-\frac{1}{2}\right) \Delta_{J} \leq J_{k}<\left(m+\frac{1}{2}\right) \Delta_{J}\right\} & \\
m & =-N_{J}^{(k)}, \cdots, N_{J}^{(k)}
\end{aligned}
$$

where $\Delta_{J}$ and $N_{J}^{(k)}$ are parameters. A good approximation is obtained if $\Delta_{J}$ is sufficiently small. Let

$$
J=\sum_{k=1, k \neq L}^{K} J_{k}
$$

The PDF of $J$ :

$\operatorname{Pr}\left\{\left(m-\frac{1}{2}\right) \Delta_{J} \leq J<\left(m+\frac{1}{2}\right) \Delta_{J}\right\}$,

$$
m=-N_{J}, \cdots, N_{J}
$$

where $N_{J}=\sum_{k=1, k \neq L}^{K} N_{J}^{(k)}$, can be obtained by convolution. From (4), the BER conditioned on $J$ is given by $Q\left(\left(2 E_{b} / N_{0}\right)^{1 / 2}(1+J)\right)$ where $Q(\cdot)$ is the standard $Q$ function. The BER conditioned on $\left(m-\frac{1}{2}\right) \Delta_{J} \leq J<\left(m+\frac{1}{2}\right) \Delta_{J}$ is therefore upper and lower bounded by

$$
P_{U}(m)=Q\left(\sqrt{2 E_{b} / N_{0}}\left(1+\left(m-\frac{1}{2}\right) \Delta_{J}\right)\right)
$$

and

$$
P_{L}(m)=Q\left(\sqrt{2 E_{b} / N_{0}}\left(1+\left(m+\frac{1}{2}\right) \Delta_{J}\right)\right)
$$

respectively. Hence, the upper $\left(P_{U}\right)$ and lower $\left(P_{L}\right)$ bounds on the average BER are

$$
\begin{aligned}
\left\{\begin{array}{l}
P_{U} \\
P_{L}
\end{array}\right\}=\sum_{m=-N_{J}}^{N_{J}} \operatorname{Pr}\left\{\left(m-\frac{1}{2}\right) \Delta_{J} \leq J<\left(m+\frac{1}{2}\right) \Delta_{J}\right\} \\
\\
\times\left\{\begin{array}{l}
P_{U}(m) \\
P_{L}(m)
\end{array}\right\} .
\end{aligned}
$$

It is apparent that the tightness of the bounds is determined by $\Delta_{J}$. By selecting a sufficiently small $\Delta_{J}$ in the computation, the bounds can be made very tight. Numerical results shown in Fig. 1 demonstrate the tightness of the bounds.

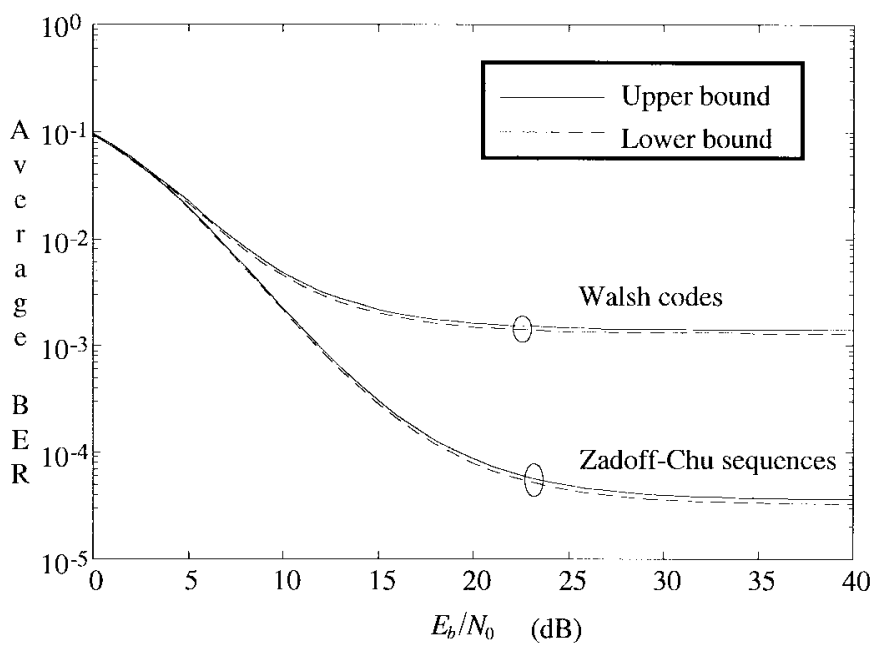

Fig. 1. Upper and lower bounds on the average BER: $K=6 ; N=32$ (Walsh codes) and $N=31$ (Zadoff-Chu sequences).

\section{CODE PERFORMANCE COMPARISON}

Properties and generation of Walsh codes have been well documented in the literature. Zadoff-Chu sequences, being a special case of generalized chirp-like sequences [9], have been proposed for asynchronous MC-CDMA [6] due to advantages of low crest factor and small maximum spectral crosscorrelation magnitude. A Zadoff-Chu sequence $\left\{a_{m}(r, q), m=0\right.$, $\cdots, N-1\}$ of length $N$ is generated by [6]

$$
a_{m}(r, q)= \begin{cases}e^{j 2 \pi(r / N)\left(m^{2} / 2+q m\right)}, & N \text { even } \\ e^{j 2 \pi(r / N)[m(m+1) / 2+q m]}, & N \text { odd }\end{cases}
$$

where $r$ is any integer relatively prime to $N$, and $q$ is any integer. If $N$ is a prime number, the set of sequences generated by $r=1, \cdots, N-1$ can be used for spreading sequences of a MC-CDMA system. The maximum number of users that can be supported is $N-1$.

We computed $P_{U}$ and $P_{L}$ for $N=32$ and $N=31$ for cases of Walsh and Zadoff-Chu sequences, respectively. In both cases, $K=6$ was assumed. Square-root-raised-cosine pulse with rolloff factor $\alpha=0.5$ was considered. Because of the assumption of spectrally nonoverlapping subcarriers, we set $\Delta f=(1+\alpha) T_{b}^{-1}$. We generated Zadoff-Chu sequences by setting $q=0$ in (12). Since we wished to compute representative $P_{U}$ and $P_{L}$ for the whole set of sequences under consideration, in obtaining an approximate PDF of $J_{k}$ we examined all combinations of spreading sequences for $k \neq L$. That is, we examined $N_{s}\left(N_{s}-1\right) / 2$ combinations of sequences where $N_{s}=32$, and 30 for cases of Walsh and Zadoff-Chu sequences, respectively.

Fig. 1 plots $P_{U}$ and $P_{L}$ against $E_{b} / N_{0}$ for the two cases of sequences. It is apparent that using Zadoff-Chu sequences gives lower average BER than using Walsh codes. Table I lists the values of $P_{U}$ and $P_{L}$ for $E_{b} / N_{0}=15 \mathrm{~dB}$. The improvement of average BER performance by using Zadoff-Chu sequences over using Walsh codes is nearly an order of magnitude. The performance improvement is quite significant.

Strictly speaking, this comparison gives the Walsh codes a slight advantage because the $K / N$ ratio for the case of 
TABLE I

UPPER $\left(P_{U}\right)$ AND LOWER $\left(P_{L}\right)$ ERRor Bounds

\begin{tabular}{c|c|c}
\multicolumn{3}{c}{ FOR $E_{b} / N_{0}=15 \mathrm{~dB}$ AND $K=6$} \\
\hline sequences & $P_{L}$ & $P_{U}$ \\
\hline Zadoff-Chu $N=31$ & $2.8 \times 10^{-4}$ & $3.0 \times 10^{-4}$ \\
Walsh $N=32$ & $2.0 \times 10^{-3}$ & $2.2 \times 10^{-3}$ \\
\hline
\end{tabular}

Walsh codes is less than that of Zadoff-Chu sequences. In the comparison under the same $K / N$ condition, performance improvement of using Zadoff-Chu sequences over Walsh codes would be even greater, and same conclusions follow.

\section{CONCLUSIONS}

We have derived upper and lower bounds on the average BER for asynchronous MC-CDMA communications using BPSK with coherent detection. The bounds can be very tight if the parameter $\Delta_{J}$ is chosen to be sufficiently small. Numerical results on the derived bounds have shown that using Zadoff-Chu sequences as spreading sequences in asynchronous MC-CDMA performs better than using Walsh codes.

\section{REFERENCES}

[1] N. Yee, J.-P. M. G. Linnartz, and G. Fettweis, "Multi-carrier CDMA in indoor wireless radio networks," IEICE Trans. Commun., vol. E77-B, pp. 900-904, July 1994.

[2] K. Fazel, S. Kaiser, and M. Schnell, "A flexible and high performance cellular communications based on orthogonal multi-carrier SSMA," Int. J. Wireless Personal Commun., pp. 121-144, 1995.

[3] K. Fazel, "Performance of CDMA/OFDM for mobile communication system," in Proc. ICUPC'93, Ottawa, Ont., Canada, Oct. 12-15, 1993. pp. 975-979.

[4] N. Yee and J.-P. Linnartz, "BER of multi-carrier CDMA in an indoor Rician fading channel," in Proc. 27th Asilomar Conf. on Signals, Systems and Computers, Pacific Grove, CA, Nov. 1-3, 1993, pp. 426-430.

[5] S. Kaiser, "OFDM-CDMA versus DS-CDMA: Performance evaluation for fading channels," in Proc. ICC'95, Seattle, WA, June 18-22, 1995, pp. $1722-1726$.

[6] B. M. Popović, "Spreading sequences for multi-carrier CDMA systems," in IEE Coll. on CDMA Techniques and Applications for Third Generation Mobile Systems, London, U.K., May 19, 1997, pp. 8/1-8/6.

[7] M. B. Pursley, "Performance evaluation for phase-coded spreadspectrum multiple-access communication-Part I: System analysis," IEEE Trans. Commun., vol. COM-25, pp. 795-799, Aug. 1977.

[8] J. S. Lehnert, "An efficient technique for evaluating direct-sequence spread-spectrum multiple-access communications," IEEE Trans. Commun., vol. 37, pp. 851-858, Aug. 1989.

[9] B. M. Popović, "Generalized chirp-like polyphase sequences with optimum correlation properties," IEEE Trans. Inform. Theory, vol. 38, pp. 1406-1409, July 1992. 